\title{
Adjunctive Aerosolized Antibiotics to Treat Ventilator-Associated Pneumonia: A Clinical Conundrum Continues
}

Ventilator-associated pneumonia (VAP) continues to be a major complication of critical illness, and patient outcomes are often suboptimal. Approximately one third of patients receiving intravenous antibiotics alone will fail therapy. In addition, an increase in multidrug-resistant strains of Pseudomonas, Acinetobacter, and enterobacteriacae (eg, carbapenemase-producing Klebsiella) limits therapeutic choices. It is not clear why so many patients fail therapy, but one factor may be that many antibiotics commonly used for VAP, such as $\beta$-lactams, aminoglycosides, and vancomycin, have relatively poor lung penetration. ${ }^{1}$ Thus, adding intratracheally administered antibiotics makes intuitive sense in an attempt to increase antibiotic concentrations at the site of infection. The term "intratracheal" encompasses all methods of delivering antibiotics directly to the lungs, but the most common practice is aerosolization (some prefer "nebulization" or "inhalation". I will use "aerosolization" generically in this editorial.)

Adding aerosolized antibiotics to intravenous antibiotics to treat severe pulmonary infections has been studied intermittently since the $1960 \mathrm{~s}^{2,3}$ However, since the turn of the millennium, there has been a sharp increase in publications in this area. Most have described aerosolized colistin or aminoglycosides as adjunctive therapy for multidrug-resistant Gram-negative VAP. Less commonly, aerosolized cephalosporins have also been used. An anticipated side benefit of this route of administration may be a decrease in systemic adverse events (such as nephrotoxicity with colistin or aminoglycosides) because aerosolization does not usually result in significant serum concentrations in patients with normal renal function..$^{2,3}$

Unfortunately, there are many unanswered questions regarding the use of aerosolized antibiotics for VAP. Most notably, the vast majority of publications to date have described retrospective, observational studies without control groups. There are no large randomized controlled tri-

\footnotetext{
Dr Wood discloses a relationship with Bayer AG and is an investigator for the INHALE 1 trial.

Correspondence: G. Christopher Wood PharmD, 881 Madison, Room 335, College of Pharmacy, University of Tennessee, Memphis, TN 38163. E-mail: cwood@uthsc.edu.
}

DOI: $10.4187 /$ respcare.05064 als comparing intravenous plus aerosolized antibiotics with intravenous therapy alone. Fortunately, some recent studies have been more rigorous (eg, using matched control

\section{See the Original Study on Page 1008}

groups). Also, an important meta-analysis of aerosolized colistin papers suggested better cure rates with its use. ${ }^{4}$ However, it is still not clear whether aerosolized antibiotics actually improve VAP outcomes.

Another important issue is making sure the administration technique effectively delivers a clinically important dose of antibiotic to the site of infection in the distal lung fields. Studies show that a number of drug formulation and administration parameters can dramatically improve the amount of drug delivered by up to 10 -fold. ${ }^{2,3}$ Some of these factors include the volume of drug solution, type and placement of the nebulizer, presence of humidification in the ventilator circuit, and ventilator model and settings. Thus, attention must be paid to proper drug preparation and administration technique. Last, the safety of aerosolized antibiotics, both in terms of adverse drug events and the impact on bacterial resistance, lacks high quality data. In particular, colistin is poorly tolerated in some patients and must be used immediately after reconstitution to avoid potentially fatal reactions.

Clearly, there are many unanswered questions regarding efficacy, safety, drug selection, dosing, drug preparation issues, and optimal administration techniques, given a bewildering array of nebulizers, ventilators, and ventilation parameters. Ideally, there should be regularly updated guidelines published every 4-5 years to guide clinicians in this uncertain and evolving area. Unfortunately, that is not happening. The American Thoracic Society/Infectious Diseases Society of America VAP guideline ${ }^{1}$ has not been updated since 2005 and did not adequately discuss aerosolized antibiotics. The Society of Infectious Diseases Pharmacists aerosolized antibiotic guideline ${ }^{2}$ did a far better job in this area, but it has not been updated since its initial publication in 2010 .

Interestingly, the current paper by Solé-Lleonart et $\mathrm{al}^{5}$ takes a step back from many of these questions and asks: How are aerosolized antibiotics actually being used in ICUs? The authors surveyed an impressive number of ICUs drawn from 6 continents. Approximately three fourths of the ICUs were in Asia and Europe, and most of the remaining sites were in North America and Australasia. There 


\section{EDITORIALS}

was also a nice distribution of medical, surgical, and trauma subjects represented. Not surprisingly to those of us who care for critically ill patients, the use of aerosolized antibiotics was common, with almost half of the surveyed ICUs using them as current practice. Use was somewhat more common in North America and Australasia than in Asia and Europe.

Perhaps a more interesting finding was the mix of concerns that clinicians had for not using aerosolized antibiotics. The lack of evidence-based guidelines and concerns about adverse events and bacterial resistance are legitimate issues that point to critical gaps in knowledge that remain. Higher quality studies, such as the currently active INHALE randomized controlled trials,,${ }^{67}$ will provide critical data in determining the efficacy and safety of aerosolized antibiotics for VAP. The other categories (lack of personal experience and lack of resources) may indirectly point to unanswered questions around drug preparation and administration issues (eg, perhaps clinicians are not using aerosolized antibiotics because they do not know how to order them, or there could be roadblocks from the pharmacy, nursing, or respiratory therapy departments). This could actually be a small opportunity to improve interprofessional teamwork. I have personally experienced this in my practice.

The second interesting finding was the type of administration used. It was shocking that $14 \%$ of ICUs used instillation instead of aerosolization. Even as a researcher and clinician in this area, I had assumed that this idea had died out in the 1970s. I guess that's why you do the survey! In a similar vein, only $9 \%$ of units were using vibrating mesh nebulizers, which are better choices than jet or ultrasonic nebulizers in terms of drug delivery and simplicity. Regarding administration technique, $>70 \%$ of the surveyed units used inadequate administration practices based on the authors' definitions. Thus, there is large opportunity to educate clinicians on using more optimal administration devices and techniques. ${ }^{5}$

The paper does have 2 important weaknesses. First, the survey topics did not cover some critical issues, such as antibiotic selection, dose, preparation, and adverse events. ${ }^{2,3}$ The authors state that a follow-up survey will be forthcoming that will address some of these issues. Second, the authors' list of accepted standard practices for aerosolized administration are debatable (and they note this in the discussion). I would argue that they left out some important issues, such as the nebulizer placement in the circuit. They also included others that were questionable, such as bronchodilator administration for every patient. ${ }^{2,3}$ This speaks to the need for more clear data on optimizing aerosolized drug administration in mechanically ventilated patients. Indeed, it is possible that ideal practices may change as the more efficient vibrating mesh nebulizers become more widely used.

Nonetheless, this paper provides a welcome and broad look into the current state of a relatively untested but widely used drug therapy for VAP. It provides additional insights into some of the gaps in knowledge that concern clinicians, and it also reinforces the need for high quality data. In the meantime, an appropriate professional organization (or organizations) should publish regularly updated guidelines on aerosolized antibiotics for VAP that include efficacy, safety, and drug administration issues.

$$
\begin{array}{r}
\text { G. Christopher Wood PharmD } \\
\text { College of Pharmacy } \\
\text { University of Tennessee Health Science Center } \\
\text { Memphis, Tennessee }
\end{array}
$$

\section{REFERENCES}

1. American Thoracic Society, Infectious Diseases Society of America. Guidelines for the management of adults with hospital-acquired, ventilator-associated, and healthcare-associated pneumonia. Am J Respir Crit Care Med 2005;171(4):388-416.

2. Le J, Ashley ED, Neuhauser MM, Brown J, Gentry C, Klepser ME, et al. Consensus summary of aerosolized antimicrobial agents: application of guideline criteria. Pharmacotherapy 2010;30(6):562-584.

3. Wood GC. Aerosolized antibiotics for treating hospital-acquired and ventilator-associated pneumonia. Expert Rev Anti Infect Ther 2011; 9(11):993-1000.

4. Valachis A, Samonis G, Kofteridis DP. The role of aerosolized colistin in the treatment of ventilator-associated pneumonia: a systematic review and metaanalysis. Crit Care Med 2015;43(3):527-533.

5. Solé-Lleonart C, Rouby JJ, Chastre J, Poulakou G, Palmer L, Blot S, et al. Intratracheal administration of antimicrobial agents in mechanically ventilated adults: an international survey on delivery practices and safety. Respir Care 2016;61(8):1008-1014.

6. Inhaled amikacin solution BAY41-6551 as adjunctive therapy in the treatment of Gram-negative pneumonia (INHALE 1). http:// clinicaltrials.gov/show/NCT01799993 Accessed June 3, 2016.

7. Inhaled amikacin solution BAY41-6551 as adjunctive therapy in the treatment of Gram-negative pneumonia (INHALE 2). http:// clinicaltrials.gov/show/NCT00805168 Accessed June 3, 2016. 